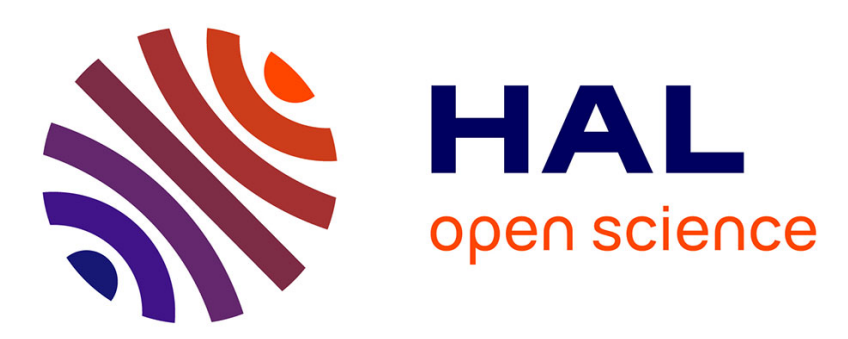

\title{
Fresnel-like formulas for the reflection and transmission of surface phonon-polaritons at a dielectric interface
}

Jose Ordonez-Miranda, Laurent Tranchant, Sergei Gluchko, Thomas Antoni, Sebastian Volz

\section{- To cite this version:}

Jose Ordonez-Miranda, Laurent Tranchant, Sergei Gluchko, Thomas Antoni, Sebastian Volz. Fresnellike formulas for the reflection and transmission of surface phonon-polaritons at a dielectric interface. Physical Review B: Condensed Matter and Materials Physics (1998-2015), 2014, pp.10. 10.1103/PhysRevB.90.155416 . hal-01134039

\section{HAL Id: hal-01134039 \\ https://hal-centralesupelec.archives-ouvertes.fr/hal-01134039}

Submitted on 24 Mar 2015

HAL is a multi-disciplinary open access archive for the deposit and dissemination of scientific research documents, whether they are published or not. The documents may come from teaching and research institutions in France or abroad, or from public or private research centers.
L'archive ouverte pluridisciplinaire HAL, est destinée au dépôt et à la diffusion de documents scientifiques de niveau recherche, publiés ou non, émanant des établissements d'enseignement et de recherche français ou étrangers, des laboratoires publics ou privés. 


\title{
Fresnel-like Formulas for the Reflection and Transmission of Surface Phonon-Polaritons at a Dielectric Interface
}

\author{
Jose Ordonez-Miranda, ${ }^{1}$ Laurent Tranchant, ${ }^{1}$ Sergei \\ Gluchko, ${ }^{1}$ Thomas Antoni, ${ }^{1,2}$ and Sebastian Volz ${ }^{1, *}$ \\ ${ }^{1}$ Laboratoire d'Énergétique Moléculaire et Macroscopique, \\ Combustion, UPR CNRS 288, École Centrale Paris, \\ Grande Voie des Vignes, 92295 Châtenay-Malabry, France. \\ ${ }^{2}$ École Centrale Paris, Laboratoire de Photonique Quantique et Moléculaire, \\ UMR CNRS 853\%, École Normale Supérieure de Cachan, \\ Grande Voie des Vignes, F-92295 Châtenay-Malabry CEDEX, France.
}

The reflection and transmission coefficients of a surface phonon-polariton propagating along the surface of a thin film of $\mathrm{SiO}_{2}$ and crossing the interface of two dielectric media are analytically determined. Based on the expansion of the electrical and magnetic fields in terms of normal modes, explicit expressions for the reflectivity and transmissivity of the radiation fields generated at the dielectric interface are also obtained. Symmetrical and simple Fresnel-like formulas are derived for nanofilms. For the dielectric interfaces of air $/ \mathrm{BaF}_{2}$ and air $/ \mathrm{Al}_{2} \mathrm{O}_{3}$, it is shown that: i) The polariton reflectivity (transmissivity) decreases (increases) as the film thickness increases, while its radiation equivalent follows the opposite behavior. ii) In the polariton and radiation fields, the transmissivity is significantly more sensitive than the reflectivity to the changes on the permittivity mismatch of the dielectric interface. For a $143 \mathrm{~nm}$-thick film, the polariton transmissivity (reflectivity) changes $13.2 \%(1.9 \%)$, when this mismatch varies by $50 \%$. iii) The reflectivity and transmissivity of the radiation fields are smaller than their polariton counterparts, which together account for around $82 \%$ of the total energy. The proposed formalism accurately fulfils the principle of conservation of energy for describing the reflection and transmission of both the polariton and radiation fields generated at a dielectric interface.

\footnotetext{
* Corresponding author: sebastian.volz@ecp.fr
} 


\section{INTRODUCTION}

Surface phonon-polartions (SPPs) have been recently proposed [1-3] as potential energy carriers to enhance significantly the phonon heat transport in polar nanomaterials with low thermal conductivity, as is the case of the amorphous $\mathrm{SiO}_{2}$ and crystalline $\mathrm{SiC}$. Many theoretical [4-7] and experimental[8,9] studies have shown that the propagation length of SPPs traveling along the interface of nanofilms can be more than three orders of magnitude larger than the corresponding phonon mean free path, which yields in turn a SPP thermal conductivity comparable or higher than the phonon counterpart. We have also shown that the thermal conductance of polar nanowires due to SPPs is quantized and its value can be comparable to the one of phonons at room temperature [10]. Furthermore, various research groups have shown that the SPPs have promising applications on the thermal performance of nanoscale devices $[4,8,11]$, radiative heat transfer [12-15], high-density infrared data storage [16], surface infrared absorption [17], coherent thermal emission [18], and photonics $[19,20]$. Given that SPPs are electromagnetic waves that propagates along the interface between polar and dielectric materials, their energy transport is expected to increase as the material size is scaled down to nanoscales, due to the predominance of the surface effects at these size scales $[4,11]$.

SPP energy transport is determined by material permittivities and hence it can be modified by material discontinuities, which can be present in optical/electronic integrated circuits printed out on surfaces supporting the propagation of SPPs. The study of the reflection and transmission of SPPs at these discontinuities is thus of great practical interest and it has attracted considerable experimental [21-23] and theoretical [24-26] interest in recent years. By

using Fourier transform infrared spectroscopy, Chen et al. [21] measured the transmissivity through a $1 \mu \mathrm{m}$-thick film of $\mathrm{SiO}_{2}$ deposited over a substrate of a $500 \mu \mathrm{m}$-thick silicon wafer and showed that the minima of the fraction of transmitted energy occur at the resonance wavelengths of absorption. For the major resonance peak, they found a fivefold increase in the transmission across a solid film through a perforated film with a periodic array of circular holes. These results are consistent with the numerical ones obtained by Catrysse and Fan [24], for the transmission through subwavelength hole arrays in a thin film of $\mathrm{SiC}$, by means of a three-dimensional 3D finite-difference time domain method. Similar experimental data were reported by Gall et al. [22] and Marquier et al. [23] for the reflectivity and 
transmissivity spectra in a $\mathrm{SiC}$ grating supporting the propagation of SPPs. Furthermore, by placing infinitely conducting metallic planes both above and below the propagation interface and making a waveguide-like structure, Stegeman et al. [25-27] numerically calculated the transmission and reflection coefficients for a surface plasmon polariton crossing a plane boundary at normal incidence. They showed that these coefficients vary strongly with the ratio between the separation distance of the metallic planes and the polariton wavelength, such that the reflection (transmission) coefficient tends to zero (unity) as this ratio increases. Even though the formalism proposed by these latter authors is rigorous and could also be extended for SPPs, their numerical calculations are quite complicated and time consuming to implement, as was mentioned by the same authors [27].

In this paper, we analytically determine the reflection and transmission coefficients of a SPP propagating along the surface of a thin film of $\mathrm{SiO}_{2}$ and crossing the interface of two dielectric media. Based on the expansion of the electrical and magnetic fields in terms of normal modes, explicit expressions for the reflection and transmission coefficients of the radiation fields generated at the dielectric interface are also derived. It is shown that these latter fields are indispensable for satisfying the boundary conditions and the principle of conservation of energy. Our results provide clear physical insights on how the SPP and radiation energy depends on the permittivity of the dielectric media as well as on the permittivity and thickness of the thin film.

\section{FORMULATION OF THE PROBLEM AND NORMAL MODES}

Let us consider a layered system supporting the propagation of a SPP, as shown in Fig. 1. The thin film of thickness $d$ and permittivity $\varepsilon_{1}(\omega)$ is a polar material (as $\mathrm{SiO}_{2}$ and $\mathrm{SiC}$ ) deposited over a metallic substrate, which limits the generation of radiation modes at the vertical interface of the semi-infinite dielectric media of permittivities $\varepsilon_{2}$ and $\varepsilon_{3}$ [28]. When the incident SPP propagating along the interface $1-2$ arrives to the vertical interface $z=0$, it is partially reflected backward and transmitted to the interface $1-3$. In general, the incident SPP can also be partially reflected and transmitted as electromagnetic radiation, which is predicted by the Maxwell equations and is required to satisfy the boundary conditions at $z=0$, as shown below. The problem consists in finding the reflection and transmission coefficients of both the SPP and radiation electromagnetic fields, which will allow us to 


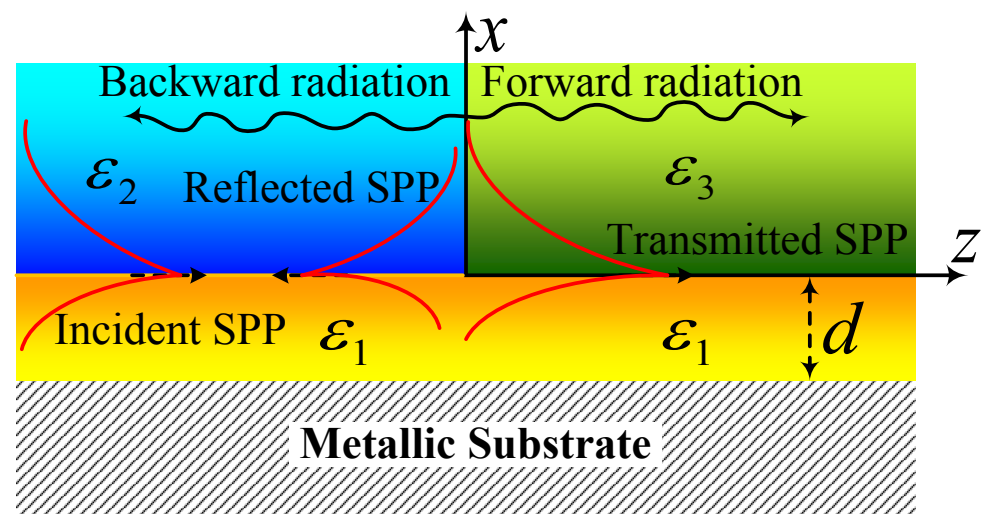

FIG. 1. SPP and radiation modes generated by an incident SPP at a dielectric interface. The metallic substrate of the film of thickness $d$ is used to diminish the radiation modes and enhance the SPP energy transport.

quantify the distribution of energy in the reflected and transmitted SPP, as well as in the radiation losses. By analogy with the problems involving waveguides, the electrical and magnetic fields for the SPP and radiation fields in each region of the system shown in Fig. 1 can be expressed in terms of the corresponding modes in absence of the discontinuity at $z=0$ $\left(\varepsilon_{2}=\varepsilon_{3}\right)$. By solving the Maxwell equations under the transverse magnetic polarization, which is required for the existence of the incident SPP [4], it can be shown that the non-zero components of these fields are given by [29]

$$
\begin{gathered}
E_{x}^{(n)}=\frac{\beta}{\omega \varepsilon} H_{y}^{(n)}, \\
E_{z}^{(n)}=\frac{1}{i \omega \varepsilon_{n}} \frac{\partial H_{y}^{(n)}}{\partial x}, \\
\frac{\partial^{2} H_{y}^{(n)}}{\partial x^{2}}-p_{n}^{2} H_{y}^{(n)}=0
\end{gathered}
$$

where $\beta$ and $p_{n}$ are the in-plane and transverse wave vectors, respectively, and they are related by $p_{n}^{2}=\beta^{2}-\varepsilon_{n} k_{0}^{2}, k_{0}=\omega / c, \omega$ is the excitation frequency, $c$ is the speed of light in vacuum, $\varepsilon_{n}$ is the permittivity of the medium $n=1,2$, and $i=\sqrt{-1}$ is the imaginary unit. The electrical $\left(E_{x, z}^{(n)}\right)$ and magnetic $\left(H_{y}^{(n)}\right)$ components depend on the coordinate $z$ and time $t$ through their common factor $\exp (i(\omega t-\beta z))$, which is omitted in Eqs. (1a)-(1c) and will be suppressed hereafter. For $\varepsilon_{3}=\varepsilon_{2}$, Eq. (1c) establishes that the magnetic field inside the film $\left(H_{y}^{(1)}\right)$ and the semi-infinite medium $\left(H_{y}^{(2)}\right)$ are given by 


$$
\begin{gathered}
H_{y}^{(1)}=A \cosh \left(p_{1}(x+d)\right), \\
H_{y}^{(2)}=B e^{p_{2} x}+C e^{-p_{2} x},
\end{gathered}
$$

where $H_{y}^{(1)}$ has been chosen in such a way that the associated electrical field $E_{x}^{(1)}$ satisfies the boundary condition $E_{x}^{(1)}(x=-d)=0$, which is imposed by the metallic substrate [30]. The parameters $A, B$, and $C$ are determined by the excitation source and the boundary conditions $H_{y}^{(1)}=H_{y}^{(2)}$ and $E_{z}^{(1)}=E_{z}^{(2)}$ at the interface $x=0$. These latter relations yield

$$
\begin{gathered}
B+C=A \cosh \left(p_{1} d\right), \\
B-C=A \alpha_{21} \sinh \left(p_{1} d\right),
\end{gathered}
$$

where $\alpha_{21}=\varepsilon_{2} p_{1} / \varepsilon_{1} p_{2}$. Equations (1)-(3) are valid for both the SPP and radiation fields, which are going to be analyzed separately, as follows.

\section{A. SPP modes}

These modes are defined by the exponential decay $\left(\operatorname{Re}\left(p_{2}\right)>0\right)$ of the fields as they travel away from the interface $x=0$. This condition requires that $B=0$, for which Eqs. (3a) and (3b) yield the following dispersion relation

$$
\frac{p_{2}}{\varepsilon_{2}}+\frac{p_{1}}{\varepsilon_{1}} \tanh \left(p_{1} d\right)=0
$$

According to Eqs. (2) and (3a), the transverse magnetic field $\left(h(x)=H_{y}\right)$ can be conveniently written as

$$
h(x)=N_{S}\left\{\begin{array}{l}
\cosh \left(p_{1}(x+d)\right),-d<x<0 \\
\cosh \left(p_{1} d\right) e^{-p_{2} x}, x>0
\end{array}\right.
$$

where $N_{S}=A$ for the SPP modes, and the transverse electrical field is given by $e(x)=$ $(\beta / \omega \varepsilon) h(x)$ (see Eq.(1a)). Equation (5) thus defines one SPP mode for each pair $\left(p_{1}, p_{2}\right)$ of transverse wave vectors that satisfy the dispersion relation in Eq. (4). According to the appendix A, these modes are orthogonal and they satisfy the normalization condition 


$$
\begin{gathered}
\int_{-d}^{\infty} e(x) h^{*}(x) d x=1 \\
N_{S}^{2}=\frac{2 \omega \varepsilon_{1}}{\beta d \gamma} \\
\gamma=1+\frac{\sinh \left(2 p_{1} d\right)}{2 p_{1} d}+\frac{\varepsilon_{1}}{\varepsilon_{2}} \frac{\cosh ^{2}\left(p_{1} d\right)}{p_{2} d},
\end{gathered}
$$

where the ${ }^{*}$ stands for the complex conjugate of $h(x)$.

\section{B. Radiation modes}

The electrical and magnetic fields of these modes are not spatially damped along the $x$ direction and therefore they are defined by $\operatorname{Re}\left(p_{2}\right)=0$. In this case, the transverse wave vector $p_{2}$ can be defined as $p_{2}=-i k$, where $k>0$ is the radiation wave vector along the transverse $x$ direction. Based on Eqs. (2) and (3) and by analogy with the SPP modes, the radiation modes can be defined as follows

$$
h(x, k)=N_{R} \begin{cases}\cosh \left(p_{1}(x+d)\right) & ,-d<x<0 \\ \cosh \left(p_{1} d\right) \cos (k x)+\xi \sinh \left(p_{1} d\right) \sin (k x) & , x>0\end{cases}
$$

where $N_{R}=A$ for the radiation modes, $\xi=\varepsilon_{2} p_{1} / \varepsilon_{1} k$, and $\beta^{2}=\varepsilon_{2} k_{0}^{2}-k^{2}=p_{1}^{2}+\varepsilon_{1} k_{0}^{2}$. The corresponding electrical field is given by $e(x, k)=(\beta / \omega \varepsilon) h(x, k)$, as before. These radiation modes are also orthogonal and they are normalized by the following condition (Appendix A)

$$
\begin{gathered}
\int_{-d}^{\infty} e(x, k) h^{*}\left(x, k^{\prime}\right) d x=\delta\left(k-k^{\prime}\right), \\
N_{R}^{2}=\frac{2 \omega \varepsilon_{2}}{\pi \beta(k) \gamma_{R}}, \\
\gamma_{R}=\left|\cosh \left(p_{1}(k) d\right)\right|^{2}+\left|\xi \sinh \left(p_{1}(k) d\right)\right|^{2} .
\end{gathered}
$$

We can now use the previous SPP and radiation modes to express the electrical and magnetic fields involved in the system shown in Fig. 1. Let $e_{0}^{<}(x)$ and $h_{0}^{<}(x)\left(e_{0}^{>}(x)\right.$ and $\left.h_{0}^{>}(x)\right)$ be the transverse components of the electrical and magnetic fields of the incident 
(transmitted) SPP, respectively; and $e^{<}(x, k)$ and $h^{<}(x, k)\left(e^{>}(x, k)\right.$ and $\left.h^{>}(x, k)\right)$ be the corresponding electromagnetic fields of the backward (forward) radiation modes. The transverse components of the total electromagnetic fields in the regions $z<0\left(E_{x}^{<}, H_{y}^{<}\right)$and $z>0$ $\left(E_{x}^{>}, H_{y}^{>}\right)$can then be expressed as follows [31]

$$
\begin{gathered}
E_{x}^{<}(x, z)=e_{0}^{<}(x)\left(e^{-i z \beta^{<}}-r e^{i z \beta^{<}}\right)-\int_{0}^{\infty} r_{R}(k) e^{<}(x, k) e^{i z \beta_{R}^{<}} d k, \\
H_{y}^{<}(x, z)=h_{0}^{<}(x)\left(e^{-i z \beta^{<}}+r e^{i z \beta^{<}}\right)+\int_{0}^{\infty} r_{R}(k) h^{<}(x, k) e^{i z \beta_{R}^{<}} d k, \\
E_{x}^{>}(x, z)=\tau e_{0}^{>}(x) e^{-i z \beta^{>}}+\int_{0}^{\infty} \tau_{R}(k) e^{>}(x, k) e^{-i z \beta_{R}^{>}} d k, \\
H_{y}^{>}(x, z)=\tau h_{0}^{>}(x) e^{-i z \beta^{<}}+\int_{0}^{\infty} \tau_{R}(k) h^{>}(x, k) e^{-i z \beta_{R}^{<}} d k,
\end{gathered}
$$

where $r$ and $\tau\left(r_{R}\right.$ and $\left.\tau_{R}\right)$ are the reflection and transmission coefficients of the SPP (radiation) fields, respectively, and $\beta^{<}$and $\beta^{>}\left(\beta_{R}^{<}\right.$and $\beta_{R}^{>}$) are the SPP (radiation) wave vectors along the propagation direction, for $z<0$ and $z>0$, respectively. The continuity of the tangential electrical $\left(E_{x}^{<}(x, 0)=E_{x}^{>}(x, 0)\right)$ and magnetic $\left(H_{y}^{<}(x, 0)=H_{y}^{>}(x, 0)\right)$ fields at the dielectric interface $z=0$ yields

$$
\begin{gathered}
(1-r) e_{0}^{<}(x)-\int_{0}^{\infty} r_{R}(k) e^{<}(x, k) d k=\tau e_{0}^{>}(x)+\int_{0}^{\infty} \tau_{R}(k) e^{>}(x, k) d k, \\
(1+r) h_{0}^{<}(x)+\int_{0}^{\infty} r_{R}(k) h^{<}(x, k) d k=\tau h_{0}^{>}(x)+\int_{0}^{\infty} \tau_{R}(k) h^{>}(x, k) d k .
\end{gathered}
$$

Note that in absence of the radiation terms, Eqs. (10a) and (10b) cannot be satisfied because of the transverse wave vectors in the region $z<0$ are different than the corresponding ones in $z>0$. It is therefore clear that the presence of the radiation modes are indispensable to satisfy these boundary conditions. This mode matching approach was previously applied to numerically determine the reflection and transmission coefficients of surface plasmon-polaritons at dielectric interfaces [32,33]. To eliminate the dependence on the variable $x$ and to solve Eqs. (10a) and (10b) for the coefficients $r, \tau, r_{R}$, and $\tau_{R}$, it is convenient to define the following inner product

$$
(f, g)=\int_{-d}^{\infty} f(x) g^{*}(x) d x
$$

where $f$ and $g$ are functions of $x$ over the interval of integration. Multiplying Eq. (10a) by $\left(h_{0}^{<}\right)^{*}$ and Eq. (10b) by $\left(e_{0}^{>}\right)^{*}$, and integrating both sides over the interval $-d<x<\infty$; 
the following expressions are found for $\tau$

$$
\begin{aligned}
& \tau=(1-r)\left(e_{0}^{<}, h_{0}^{>}\right)-\int_{0}^{\infty} r_{R}(k)\left(e^{<}(k), h_{0}^{>}\right) d k \\
& \tau=(1+r)\left(h_{0}^{<}, e_{0}^{>}\right)+\int_{0}^{\infty} r_{R}(k)\left(h^{<}(k), e_{0}^{>}\right) d k
\end{aligned}
$$

where we have used the normalization condition in Eq.(6a) for the SPP modes and the orthogonality of the SPP and radiation modes, which is discussed in appendix A. By following a similar procedure, it is easy to show that $\tau_{R}$ is determined by

$$
\begin{aligned}
& \tau_{R}(k)=(1-r)\left(e_{0}^{<}, h^{>}(k)\right)-\int_{0}^{\infty} r_{R}\left(k^{\prime}\right)\left(e^{<}\left(k^{\prime}\right), h^{>}(k)\right) d k^{\prime}, \\
& \tau_{R}(k)=(1+r)\left(h_{0}^{<}, e^{>}(k)\right)+\int_{0}^{\infty} r_{R}\left(k^{\prime}\right)\left(h^{<}\left(k^{\prime}\right), e^{>}(k)\right) d k^{\prime} .
\end{aligned}
$$

Equations (12) and (13) show that the exact determination of the reflection and transmission coefficients is quite complicated due to the presence of the integral terms. However, for dielectric media with permittivities $\varepsilon_{2}$ and $\varepsilon_{3}$ of the same order of magnitude, both reflection coefficients are expected to be close to zero $\left(r, r_{R} \rightarrow 0\right)$, and the electromagnetic modes in the regions $z<0$ and $z>0$ are approximately orthogonal $\left[\left(e^{<}(k), h_{0}^{>}\right),\left(h^{<}(k), e_{0}^{>}\right),\left(e_{0}^{<}, h^{>}(k)\right),\left(h_{0}^{<}, e^{>}(k)\right)\right] \rightarrow 0$ and $\left(e^{<}\left(k^{\prime}\right), h^{>}(k)\right) \approx \delta\left(k-k^{\prime}\right)$. Under these conditions and a first-order approximation of Eqs. (12) and (13), it is found that the reflection and transmission coefficients of the SPP and radiation modes are analytically given by

$$
\begin{aligned}
r & =\frac{\left(e_{0}^{<}, h_{0}^{>}\right)-\left(h_{0}^{<}, e_{0}^{>}\right)}{\left(e_{0}^{<}, h_{0}^{>}\right)+\left(h_{0}^{<}, e_{0}^{>}\right)}, \\
\tau & =\frac{2\left(e_{0}^{<}, h_{0}^{>}\right)\left(h_{0}^{<}, e_{0}^{>}\right)}{\left(e_{0}^{<}, h_{0}^{>}\right)+\left(h_{0}^{<}, e_{0}^{>}\right)}, \\
2 r_{R}(k) & =\left(e_{0}^{<}, h^{>}(k)\right)-\left(h_{0}^{<}, e^{>}(k)\right), \\
2 \tau_{R}(k) & =\left(e_{0}^{<}, h^{>}(k)\right)+\left(h_{0}^{<}, e^{>}(k)\right) .
\end{aligned}
$$

Note that while the SPP reflection and transmission coefficients depend only on inner products of SPP modes, their radiation counterparts are determined by inner products between the SPP modes in $z<0$ with radiation modes in $z>0$. This means that the origin of the radiation fields is the incident SPP field, as expected. After evaluating the inner products 
in Eqs. (14a)-(14d) with the help of Eqs. (5)-(8) and (11), the reflection and transmission coefficients can be written explicitly as follows

$$
\begin{gathered}
r=\frac{\varepsilon_{2} \varepsilon_{3}\left(\beta^{<}-\beta^{>}\right) I_{1}+\varepsilon_{1}\left(\varepsilon_{3} \beta^{<}-\varepsilon_{2} \beta^{>}\right) I_{2}}{\varepsilon_{2} \varepsilon_{3}\left(\beta^{<}+\beta^{>}\right) I_{1}+\varepsilon_{1}\left(\varepsilon_{3} \beta^{<}+\varepsilon_{2} \beta^{>}\right) I_{2}}, \\
\tau=\sigma \frac{\left(\varepsilon_{2} I_{1}+\varepsilon_{1} I_{2}\right)\left(\varepsilon_{3} I_{1}+\varepsilon_{1} I_{2}\right)}{\varepsilon_{2} \varepsilon_{3}\left(\beta^{<}+\beta^{>}\right) I_{1}+\varepsilon_{1}\left(\varepsilon_{3} \beta^{<}+\varepsilon_{2} \beta^{>}\right) I_{2}}, \\
r_{R}=\sigma\left[\left(\beta^{<}-\left(\beta_{R}^{>}\right)^{*}\right) \frac{J_{1}}{\varepsilon_{1}}+\left(\frac{\beta^{<}}{\varepsilon_{2}}-\frac{\left(\beta_{R}^{>}\right)^{*}}{\varepsilon_{3}}\right) J_{2}\right], \\
\tau_{R}=\sigma\left[\left(\beta^{<}+\left(\beta_{R}^{>}\right)^{*}\right) \frac{J_{1}}{\varepsilon_{1}}+\left(\frac{\beta^{<}}{\varepsilon_{2}}+\frac{\left(\beta_{R}^{>}\right)^{*}}{\varepsilon_{3}}\right) J_{2}\right],
\end{gathered}
$$

where the parameters $\sigma=4 \sqrt{\beta^{<} \beta^{>} / \delta^{<} \delta^{>}}, \sigma_{R}=\sqrt{\varepsilon_{1} \varepsilon_{3} d /\left(\beta^{<}\left(\beta_{R}^{>}\right)^{*} \delta^{<} \eta^{>}\right)}$, and the dimentionless integrals

$$
\begin{gathered}
I_{1}=\frac{1}{2}\left[\frac{\sinh \left[\left(p_{1}^{<}+p_{1}^{>}\right) d\right]}{\left(p_{1}^{<}+p_{1}^{>}\right) d}+\frac{\sinh \left[\left(p_{1}^{<}-p_{1}^{>}\right) d\right]}{\left(p_{1}^{<}-p_{1}^{>}\right) d}\right], \\
I_{2}=\frac{\cosh \left(p_{1}^{<} d\right) \cosh \left(p_{1}^{>} d\right)}{\left(p_{2}+p_{3}\right) d}, \\
J_{1}=\frac{1}{2}\left[\frac{\sinh \left[\left(p_{1}^{<}+\left(p_{1 R}^{>}\right)^{*}\right) d\right]}{\left(p_{1}^{<}+\left(p_{1 R}^{>}\right)^{*}\right) d}+\frac{\sinh \left[\left(p_{1}^{<}-\left(p_{1 R}^{>}\right)^{*}\right) d\right]}{\left(p_{1}^{<}-\left(p_{1 R}^{>}\right)^{*}\right) d}\right], \\
J_{2}=\frac{\cosh \left(p_{1}^{<} d\right)}{\left(p_{2}^{2}+k^{2}\right) d}\left[p_{2} \cosh \left(\left(p_{1 R}^{>}\right)^{*} d\right)+\frac{\varepsilon_{3}}{\varepsilon_{1}}\left(p_{1 R}^{>}\right)^{*} \sinh \left(\left(p_{1 R}^{>}\right)^{*} d\right)\right] .
\end{gathered}
$$

Equations (15a)-(15d) clearly show that the reflection and transmission coefficients for the SPP and radiation fields depend strongly on the permittivity mismatch of the semi-infinite media, as well as on the permittivity and thickness of the thin film. In absence of the dielectric interface $\left(\varepsilon_{3}=\varepsilon_{2}\right)$, the in-plane wave vectors $\beta^{<}=\beta^{>}$and therefore $r=0, \tau=1$, and $r_{R}=\tau_{R}=0$, as expected. For very thin $\left(k_{0} d<<1\right)$ and very thick $\left(k_{0} d>>1\right)$ films, Eqs. (15a) and (15b) becomes independent of the film thickness $d$ and they can be significantly simplified. In the first limiting case, Eqs. (15a) and (15b) reduce to

$$
\begin{gathered}
r_{0}=\frac{\sqrt{\varepsilon_{3}}-\sqrt{\varepsilon_{2}}}{\sqrt{\varepsilon_{3}}+\sqrt{\varepsilon_{2}}}, \\
\tau_{0}=4 \frac{\sqrt[4]{\varepsilon_{2} \varepsilon_{3}}}{\sqrt{\varepsilon_{2}}+\sqrt{\varepsilon_{3}}} \frac{\sqrt{\varepsilon_{2} \varepsilon_{3}\left(\varepsilon_{2}-\varepsilon_{1}\right)\left(\varepsilon_{3}-\varepsilon_{1}\right)}}{\varepsilon_{2}\left(\varepsilon_{2}-\varepsilon_{1}\right)+\varepsilon_{3}\left(\varepsilon_{3}-\varepsilon_{1}\right)},
\end{gathered}
$$

where the subscript "0" indicates that the formulas are valid for $\left(k_{0} d<<1\right)$. For thick films, 
on the other hand, Eqs.(15a) and (15b) take the form

$$
\begin{gathered}
r_{\infty}=\frac{\varepsilon_{2} \varepsilon_{3}\left(\beta^{<}-\beta^{>}\right)+\varepsilon_{1}\left(\varepsilon_{3} \beta^{<}-\varepsilon_{2} \beta^{>}\right) \chi}{\varepsilon_{2} \varepsilon_{3}\left(\beta^{<}+\beta^{>}\right)+\varepsilon_{1}\left(\varepsilon_{3} \beta^{<}+\varepsilon_{2} \beta^{>}\right) \chi}, \\
\tau_{\infty}=\psi \frac{\left(\varepsilon_{2}+\varepsilon_{1} \chi\right)\left(\varepsilon_{3}+\varepsilon_{1} \chi\right)}{\varepsilon_{2} \varepsilon_{3}\left(\beta^{<}+\beta^{>}\right)+\varepsilon_{1}\left(\varepsilon_{3} \beta^{<}+\varepsilon_{2} \beta^{>}\right) \chi}, \\
\chi=-\frac{\varepsilon_{1}\left(p_{1}^{<}+p_{1}^{>}\right)}{\varepsilon_{2} p_{1}^{<}+\varepsilon_{3} p_{1}^{>}} \\
\psi=-\frac{4 \varepsilon_{2} \varepsilon_{3} \sqrt{\beta^{<} \beta^{>} p_{1}^{<} p_{1}^{>}}}{\left(p_{1}^{<}+p_{1}^{>}\right) \sqrt{\left(\varepsilon_{1}^{2}-\varepsilon_{2}^{2}\right)\left(\varepsilon_{1}^{2}-\varepsilon_{3}^{2}\right)}},
\end{gathered}
$$

where $\beta^{<}=k_{0} \sqrt{\varepsilon_{1} \varepsilon_{2} /\left(\varepsilon_{1}+\varepsilon_{2}\right)}, \quad \beta^{>}=k_{0} \sqrt{\varepsilon_{1} \varepsilon_{3} /\left(\varepsilon_{1}+\varepsilon_{3}\right)}, p_{1}^{<}=\sqrt{-\varepsilon_{1} / \varepsilon_{2}} \beta^{<}, p_{1}^{>}=$ $\sqrt{-\varepsilon_{1} / \varepsilon_{3}} \beta^{>}$, and the subscript " $\infty$ " stands for $k_{0} d>>1$. Note that both $r_{0}\left(\varepsilon_{2}, \varepsilon_{3}\right)=$ $-r_{0}\left(\varepsilon_{3}, \varepsilon_{2}\right)$ and $r_{\infty}\left(\varepsilon_{2}, \varepsilon_{3}\right)=-r_{\infty}\left(\varepsilon_{3}, \varepsilon_{2}\right)$, which means that when the semi-infinite media are interchanged, the reflection coefficient of the reflected SPP changes by a phase reversal only. This behavior coincides with the one exhibited by the corresponding Fresnel equation for normal incidence of plane waves [30]. By contrast, this phase reversal is not present in the transmission coefficient, which is invariant under the interchange of the semi-infinite media $\left(\tau_{0}\left(\varepsilon_{2}, \varepsilon_{3}\right)=\tau_{0}\left(\varepsilon_{3}, \varepsilon_{2}\right)\right.$ and $\left.\tau_{\infty}\left(\varepsilon_{2}, \varepsilon_{3}\right)=\tau_{\infty}\left(\varepsilon_{3}, \varepsilon_{2}\right)\right)$. Given that the energy fractions of the reflected and transmitted SPP are given by the square of the magnitude of $r$ and $\tau$, respectively; as shown below, the aforementioned features of the SPP reflection and transmission coefficients establish that the energy of the reflected and transmitted SPP keeps invariant under the interchange of the dielectric media. This feature is exclusive of SPPS and is not present in the radiation fields, as indicated by Eqs. (15c) and (15d).

The accuracy of the simple and approximate expressions for the reflection and transmission coefficients in Eqs. (14a)-(14c) can be evaluated by calculating the integrals in Eqs. (12) and (13) with the estimated value of the refleccion coefficient of the radiation modes in Eq. (14c). In doing this, the following expressions for the differences $\Delta r, \Delta \tau$, and $\Delta \tau_{R}(k)$ between the exact expressions of the reflection and transmission coefficients (Eqs. (12) and (13)), and their corresponding approximate results (Eqs. (14a), (14b), and (14c)); 
are obtained

$$
\begin{gathered}
\Delta r=-\frac{\int_{0}^{\infty} r_{R}(k)\left[\left(e^{<}(k), h_{0}^{>}\right)+\left(h^{<}(k), e_{0}^{>}\right)\right] d k}{\left(e_{0}^{<}, h_{0}^{>}\right)+\left(h_{0}^{<}, e_{0}^{>}\right)}, \\
\Delta \tau=\frac{\int_{0}^{\infty} r_{R}(k)\left[\left(e_{0}^{<}, h_{0}^{>}\right)\left(h^{<}(k), e_{0}^{>}\right)-\left(h_{0}^{<}, e_{0}^{>}\right)\left(e^{<}(k), h_{0}^{>}\right)\right] d k}{\left(e_{0}^{<}, h_{0}^{>}\right)+\left(h_{0}^{<}, e_{0}^{>}\right)}, \\
\Delta \tau_{R}(k)=\frac{1}{2} \int_{0}^{\infty} r_{R}\left(k^{\prime}\right)\left[\left(h^{<}\left(k^{\prime}\right), e^{>}(k)\right)-\left(e^{<}\left(k^{\prime}\right), h^{>}(k)\right)\right] d k^{\prime} .
\end{gathered}
$$

Given the complicate dependence of $r_{R}(k)$ on the wave vector $k$ (Eq. (15c)), the integrals in Eqs. (19a)-(19c) cannot be evaluated analytically. For very thin film $\left(k_{0} d<<1\right)$, which is the case of major interest to enhance the propagation of SPPs $[4,11]$, these integrals simplify significantly and the deviations exhibit the following behavior

$$
\begin{gathered}
\Delta r \text { and } \Delta \tau \propto \sqrt{\left(\varepsilon_{2}-\varepsilon_{1}\right)\left(\varepsilon_{3}-\varepsilon_{1}\right)}\left(k_{0} d\right)^{3} \\
\Delta \tau_{R}(k) \propto \sqrt{\left(\varepsilon_{2}-\varepsilon_{1}\right)}\left(k_{0} d\right)^{2},
\end{gathered}
$$

which explicitly indicate that for $k_{0} d<<1$, our approximate results in Eqs. (14a)-(14d) are accurate and their accuracy is expected to increase as the film thickness decreases. The fact that the SPP coefficients are proportional to the third power of the film thickness, while radiation one is proportional to the second power of this thickness; shows that the approximate expression of the reflection and transmission coefficients of the SPP modes become more accurate than the transmission coefficient of the radiation modes, as the film thickness is scaled down.

The power flow per unit film width of the reflected and transmitted SPP and radiation fields can now be calculated through the Poynting vector $S$ along the propagation direction ( $z$ axis), which is defined by

$$
S=\frac{1}{2} \operatorname{Re}\left(\int_{-d}^{\infty} E_{x}(x, 0) H_{y}^{*}(x, 0) d x\right),
$$

Based on Eqs. (9) and (21), we can determine the power of the incident $\left(S^{\text {inc }}\right)$, reflected $\left(S^{\text {ref }}\right)$, and transmitted $\left(S^{\text {tran }}\right)$ SPP; as well as the power of the reflected $\left(S_{R}^{\text {ref }}\right)$ and transmitted $\left(S_{R}^{\operatorname{tran}}\right)$ radiation fields. The reflectivity and transmissivity of the SPP and radiation fields are therefore defined as $R=-S^{\text {ref }} / S^{\text {inc }}, T=S^{\text {tran }} / S^{\text {inc }}, R_{R}=-S_{R}^{\text {ref }} / S^{\text {inc }}$, and $T_{R}=S_{R}^{\operatorname{tran}} / S^{\text {inc }}$. In terms of the reflection and transmission coefficients, these relations yield 


$$
\begin{gathered}
R=|r|^{2}, \\
T=|\tau|^{2}, \\
R_{R}=\int_{0}^{\infty}\left|r_{R}(k)\right|^{2} d k, \\
T_{R}=\int_{0}^{\infty}\left|\tau_{R}(k)\right|^{2} d k .
\end{gathered}
$$

Given that all the energy of the incident SPP is transfered to the reflected and transmitted SPP and radiation fields, the principle of conservation of energy establishes that

$$
R+T+R_{R}+T_{R}=1
$$

We will show below that the approximate, simple, and analytical results for the reflection and transmission coefficients involved in Eqs. (22a)-(22d) are able to satisfy Eq. (23) with an error less than $2 \%$.

\section{RESULTS AND DISCUSSIONS}

The reflectivity and transmissivity of the SPP and radiation fields generated by an incident SPP crossing the dielectric interface shown in Fig. 1 are quantified and analyzed in this section. Special emphasis is put on the effects of the film thickness and permittivities of the dielectric media. We consider a film of amorphous $\mathrm{SiO}_{2}$ (silica), which is an abundant polar material in nature and widely used in electronic applications [1, 4]. Furthermore, $\mathrm{SiO}_{2}$ is commonly employed in the manufacturing of electrical circuits, in which thermal sources generated by Joule effect might be cooled via SPPs. For the dielectric media, we are going to use air, as a natural surrounding medium, in contact with $\mathrm{BaF}_{2}$ or $\mathrm{Al}_{2} \mathrm{O}_{3}$ (alumina), which are transparent crystals in a wide range of frequencies [34] and are used in many applications related to optics, infrared spectroscopy, and electronics. All of these materials behave as lossless materials (with real permittivities) at the frequency $\omega=210 \cdot 10^{12} \mathrm{rad} / \mathrm{s}$ $\left(k_{0}=0.7 \mu \mathrm{m}^{-1}\right)$, which is going to be used in this work, to guaranty the orthogonality of the SPP and radiation modes and to simplify the energy distribution analysis based on the Poynting vector, as discussed in appendix A.

Figure 2 shows the film-thickness dependence of the wave vector $\beta$ along the propagation direction of a SPP traveling via the interface of a film of $\mathrm{SiO}_{2}$ and a dielectric medium, 


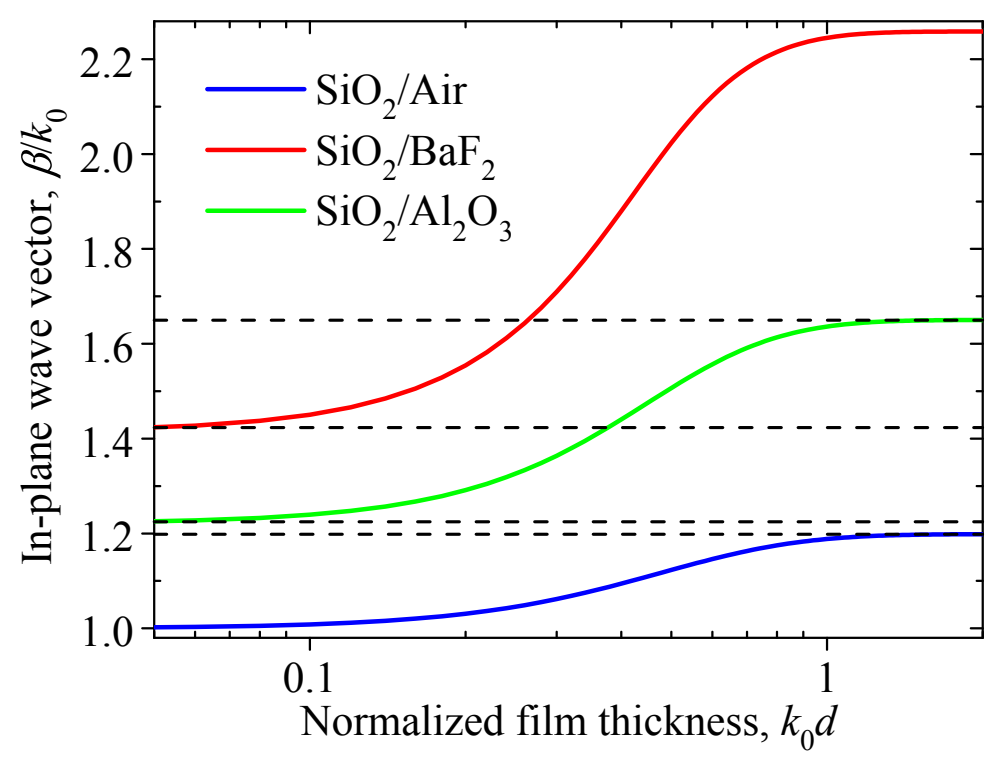

FIG. 2. Normalized in-plane wave vector of a SPP propagating along the interface of a thin film of $\mathrm{SiO}_{2}$ and a dielectric medium, as a function of the normalized film thickness. Calculations were performed for $\varepsilon(\mathrm{Air})=1, \varepsilon\left(\mathrm{BaF}_{2}\right)=2, \varepsilon\left(\mathrm{Al}_{2} \mathrm{O}_{3}\right)=1.5$, and $\varepsilon\left(\mathrm{SiO}_{2}\right)=-3.3$; which occur at $k_{0}=0.7$ $\mu \mathrm{m}^{-1}[11,34]$.

as shown in Fig. 1. Calculations were done using the dispersion relation in Eq. (4) and its analysis in Appendix B. It is seen that $\beta$ inscreases with the film thickness, such that it reaches asymptotic values for very thin $\left(k_{0} d<<1\right)$ and very thick $\left(k_{0} d>>1\right)$ films. The thick-film limit is given by the well known dispersion relation $\beta=\sqrt{\varepsilon_{1} \varepsilon_{2} /\left(\varepsilon_{1}+\varepsilon_{2}\right)} k_{0}$, and the thin-film one is described by the simple solution of the dispersion relation in Eq. (29). Given that $k_{0}=0.7 \mu \mathrm{m}^{-1}$, this latter limit holds for films with thicknesses $(d<<1.4$ $\mu \mathrm{m})$ from hundreds of nanometers downwards, which represent the regime of major energy transport by SPPs $[6,7,11]$. Note that irrespective of the the film thickness, the in-plane wave vector increases with the permittivity of the dielectric media and it is greater than the one in vacuum $\left(\beta>k_{0}\right)$, which indicates that the SPP is propagating with a wave vector to the right of the light line [11]. Furthermore, it is clear that $\beta \rightarrow k_{0}$, when the thickness and/or the dielectric permittivity reduces, which implies a photon-like nature of the SPP. As these parameters increase, $\beta / k_{0}$ separates from the unity and tends to a phonon-like behavior.

The film-thickness dependence of the normalized transverse wave vectors in the regions $z<0\left(p_{1}^{<}, p_{2}\right)$ and $z>0\left(p_{1}^{>}, p_{3}\right)$ are shown in Fig. 3, for the air $/ \mathrm{BaF}_{2}$ dielectric interface. 


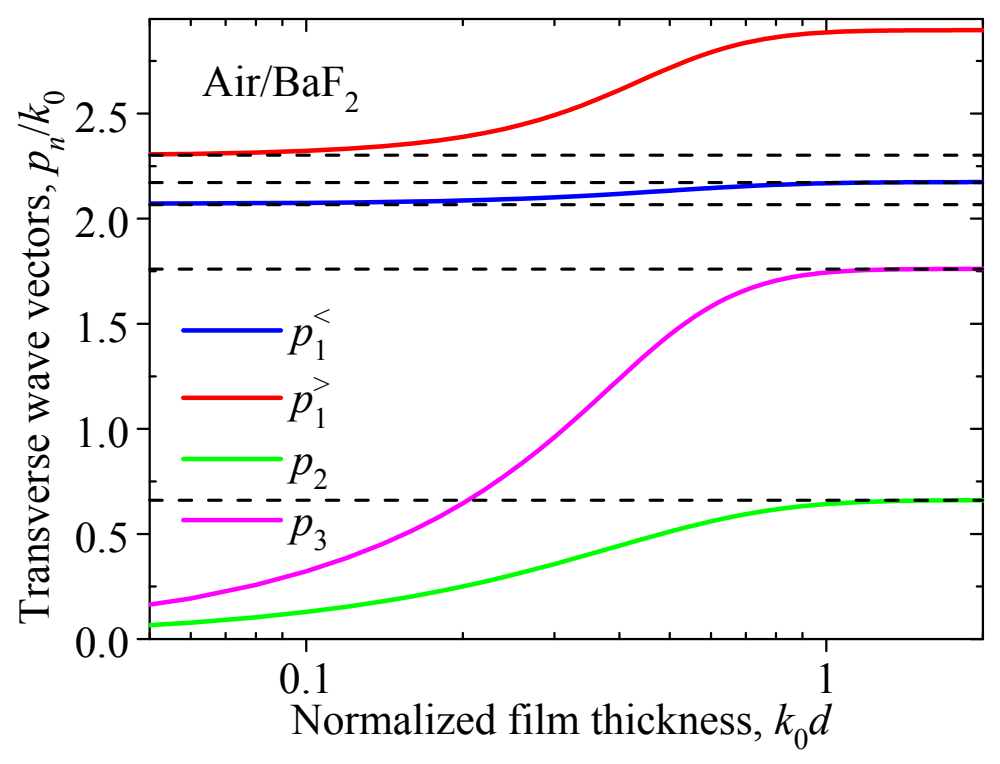

FIG. 3. Normalized transverse wave vector of a SPP propagating along the surface of a $\mathrm{SiO}_{2}$ film and crossing a dielectric interface of air $/ \mathrm{BaF}_{2}$ (see Fig. 1), as a function of the normalized film thickness. Calculations were performed with the data used in Fig. 2.

As in the case of the in-plane wave vector $\beta$, the transverse wave vectors increase with the film thickness and they become independent of this thickness for very thin $\left(k_{0} d<<1\right)$ and very thick $\left(k_{0} d>>1\right)$ films. For a given film thickness, these wave vectors are larger in the medium with higher absolute value of permittivity, as established by the dispersion relation in Eq. (4) and its analytical solution in Eq. (29). The fact that all transverse wave vectors are positive indicates that the electrical and magnetic fields of the SPPs decay spatially as they travel away from the propagation interface $x=0$, which guaranties the existence of SPPs $[4,11]$. Given that $\left|\varepsilon\left(\mathrm{SiO}_{2}\right)\right|>\varepsilon\left(\mathrm{BaF}_{2}\right)>\varepsilon($ air $)$, the transverse spatial attenuation inside the thin film is stronger than the one in $\mathrm{BaF}_{2}$, which in turns is more intense than the one within air. It is therefore clear that the SPP fields within the $\mathrm{SiO}_{2}$ film are more confined to the propagation interface than those in the dielectric media.

Figure 4 shows that reflectivity $R$ and transmissivity $T$ of the SPP fields as a function of the normalized film thickness and for two dielectric interfaces. For both cases, $R$ decreases as the film thickness increases, while $T$ exhibits the oposite trend; and they tend to thickness independent values for very thin $\left(k_{0} d<<1\right)$ and very thick $\left(k_{0} d>>1\right)$ films, which is consistent with the behavior of the SPP wave vectors shown in Figs. 2 and 3. For nanofilms, $R$ and $T$ are described by the simple and symmetrical formulas in Eqs. (17) and (20), while 


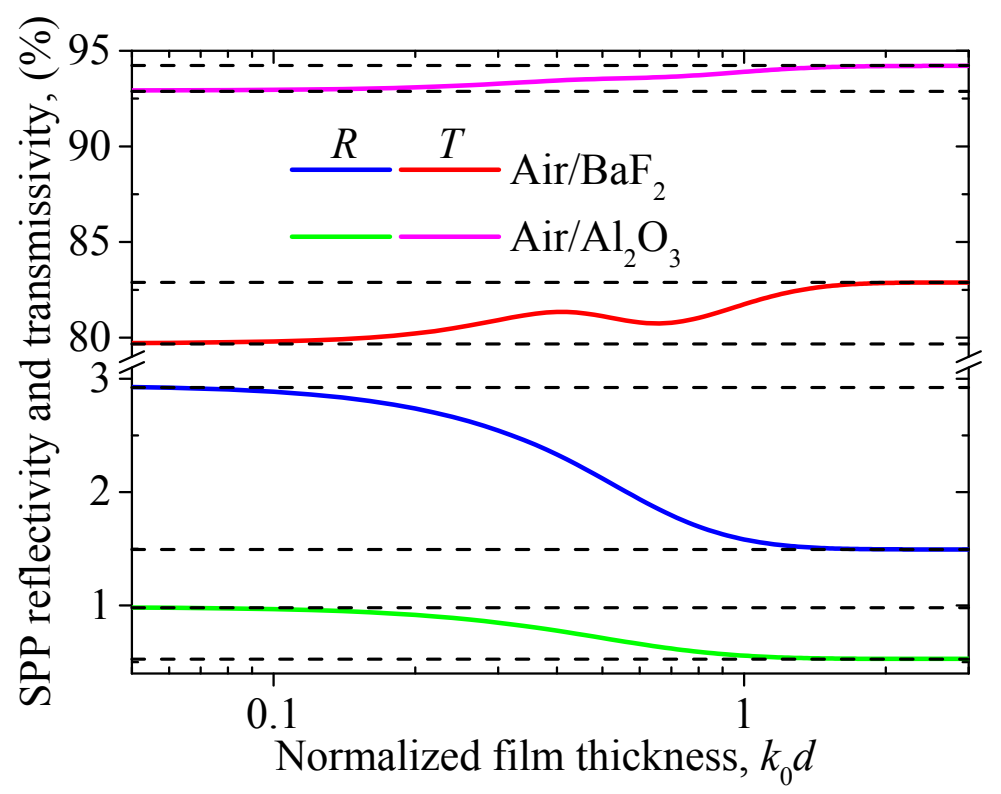

FIG. 4. Reflectivity and transmissivity of a SPP propagating along the surface of a $\mathrm{SiO}_{2}$ film and crossing a dielectric interface, as a function of the normalized film thickness. Calculations were done for two dielectric interfaces of $\mathrm{Air} / \mathrm{BaF}_{2}$ and $\mathrm{Air} / \mathrm{Al}_{2} \mathrm{O}_{3}$, and with the data used in Fig. 2 .

for micro-films, they are determined by a little more complicated but still analytical formulas in Eqs. (18) and (20). Equations (17) and (18) can thus be considered as the Fresnellike equations for the reflection and transmission of SPPs across the dielectric interface shown in Fig. 1. Note that the reflectivity (transmissivity) for the dielectric interface of $\mathrm{Air} / \mathrm{BaF}_{2}$ is higher (lower) than the corresponding one of the interface $\mathrm{Air} / \mathrm{Al}_{2} \mathrm{O}_{3}$. This is quite reasonable due to the fact that the permittivity mismatch between the dielectric media in the Air $/ \mathrm{BaF}_{2}$ interface $\left(\varepsilon_{3}-\varepsilon_{2}=1\right)$ is higher than that for the another interface $\left(\varepsilon_{3}-\varepsilon_{2}=0.5\right)$. For this modification on the dielectric interface $\left(\Delta \varepsilon_{3}=0.5\right)$ and $k_{0} d=0.1$ $(d=143 \mathrm{~nm})$, the change on the reflectivity is $\Delta R=1.9 \%$, which rises to $\Delta T=13.2 \%$ for the transmissivity. The SPP transmissivity is therefore more sensitive than the SPP reflectivity to the changes on the permittivity mismatch of the dielectric interface.

The magnitudes $|\Delta r|$ and $|\Delta \tau|$ of the deviations defined in Eqs. (19a) and (19b) for the reflection and transmission coefficients of the SPP modes are shown in Fig. 5, as a function of the normalized film thickness. For thin $\left(k_{0} d<<1\right)$ and thick $\left(k_{0} d>>1\right)$ films, both $|\Delta r|$ and $|\Delta \tau|$ becomes independent of the film thickness and they take smaller values for thinner films. This is consistent with Eq. (20a) and the predictions of the principle of conservation of 


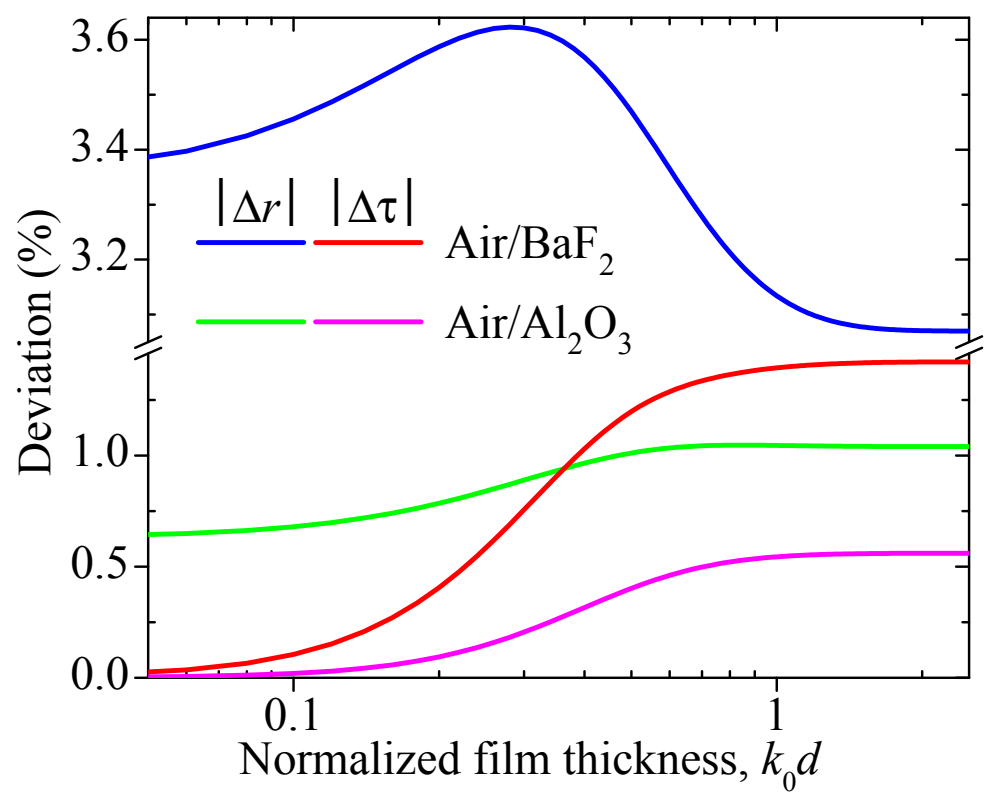

FIG. 5. Deviations of the reflection and transmission coefficients of the SPP modes propagating along the surface of a $\mathrm{SiO}_{2}$ film and crossing a dielectric interface, as a function of the normalized film thickness. Calculations were done for two dielectric interfaces of $\mathrm{Air} / \mathrm{BaF}_{2}$ and $\mathrm{Air} / \mathrm{Al}_{2} \mathrm{O}_{3}$, and with the data used in Fig. 2.

energy shown below in Fig. 8. The deviations of the reflection and transmission coefficients for the Air $/ \mathrm{BaF}_{2}$ interface are greater than their corresponding ones for the $\mathrm{Air} / \mathrm{Al}_{2} \mathrm{O}_{3}$ interface, due to the fact that the permittivity mismatch of the first interface $\left(\varepsilon_{3}-\varepsilon_{2}=1\right)$ is greater than that of the latter one $\left(\varepsilon_{3}-\varepsilon_{2}=0.5\right)$. This confirms that Eqs. (14a)-(14b) becomes more accurate as the difference of permittivities of the dielectric media reduces. Furthermore, the fact that $|\Delta \tau|<|\Delta r|<3.6 \%$ for both dielectric interfaces, indicates that the reflectivity and transmissivity shown in Fig. 4 have high accuracy, with a slight deviation from their corresponding exact values comparable or smaller than 3.6\%, as established by Eqs. (22a) and (22b).

The spectra of the reflectivity and transmissivity of the radiation fields generated by the incident SPP at the dielectric interface Air/BaF 2 are shown in Figs. 6(a) and 6(b), respectively, as a function of the normalized radiation wave vector $k / k_{0}$. Both spectra follow a similar behavior, which does not vary significantly for film thicknesses $k_{0} d>1$ and $k / k_{0}<$ $\sqrt{\varepsilon_{3}}$. Within this range of the radiation wave vector, the wave vector $\beta_{R}^{>}$is real, as established just below Eq. (7a); while for $k / k_{0}>\sqrt{\varepsilon_{3}}, \beta_{R}^{>}$becomes an imaginary number, which leads 

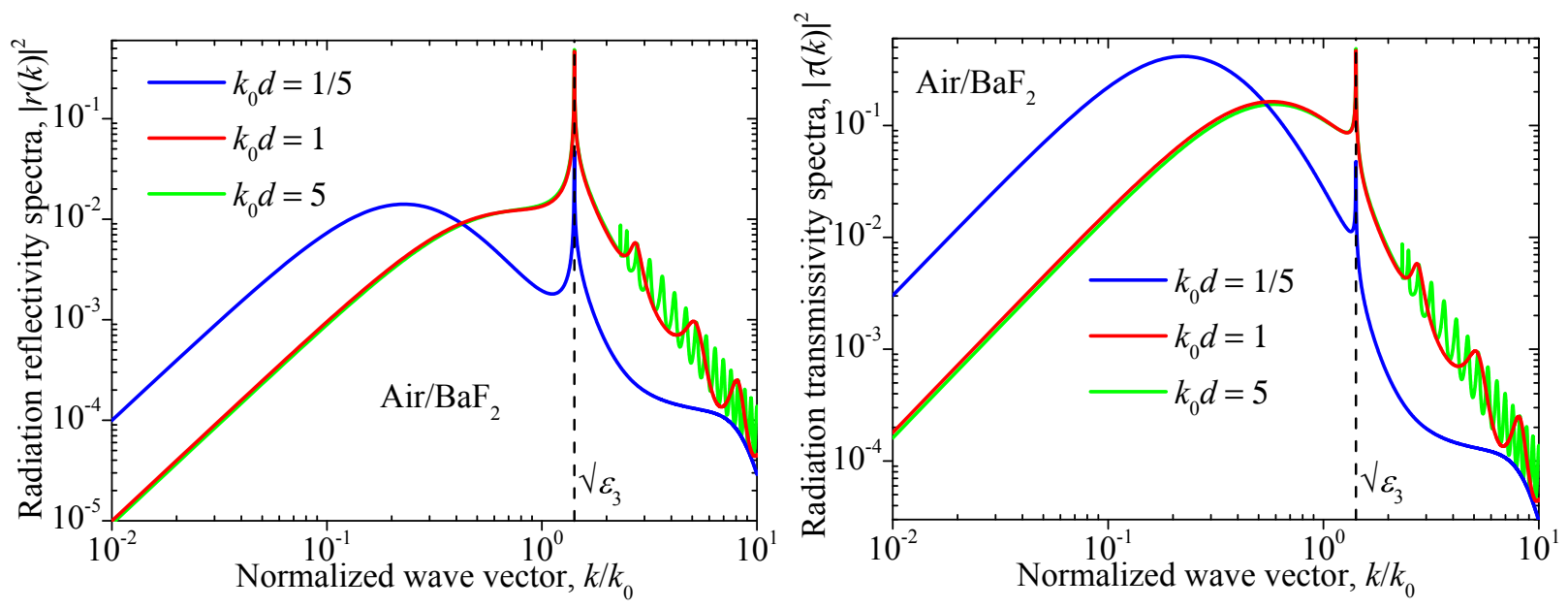

FIG. 6. Spectra of the (a) radiation reflectivity and (b) radiation transmissivity as a function of the normalized radiation wave vector. Calculations were performed for three thicknesses of a film of $\mathrm{SiO}_{2}$ and a dielectric interface of $\mathrm{Air} / \mathrm{BaF}_{2}$.

to the oscillations of the spectra. In this latter interval of the radiation wave vector, both spectra decay fast to zero as $k / k_{0}$ increases; which indicates that the major contributions to $R_{R}$ and $T_{R}$ (area under the curves) arise from wave vectors within the interval $k / k_{0}<\sqrt{\varepsilon_{3}}$. Note that, for a given film thickness, the values of the transmissivity spectrum are higher than those of the reflectivity one, and hence the transmissivity is espected to be higher than the reflectivity $\left(T_{R}>R_{R}\right)$. This is confirmed by Fig.7, not only for the Air/BaF 2 dielectric interface but also for the $\mathrm{Air} / \mathrm{Al}_{2} \mathrm{O}_{3}$ one. For both interfaces, the radiation reflectivity (transmissivity) increases (decreases) as the film thickness increases, which is opposite to the behavior exhibited by the SPP counterpart shown in Fig. 4. This is reasonable given that the energy of the incident SPP must be conserved. Both $R_{R}$ and $T_{R}$ increase as the permittivity mismatch of the dielectric interface increases from $\varepsilon_{3}-\varepsilon_{2}=1 / 2$, (for Air $/ \mathrm{Al}_{2} \mathrm{O}_{3}$ ) to $\varepsilon_{3}-\varepsilon_{2}=1$ (for Air $/ \mathrm{BaF}_{2}$ ), as expected. For this change of the dielectric interface $\left(\Delta \varepsilon_{3}=0.5\right)$ and $k_{0} d=0.1(d=143 \mathrm{~nm})$, the variation of the reflectivity is $\Delta R_{R}=0.5 \%$, which rises to $\Delta T_{R}=12.2 \%$ for the transmissivity. These changes are smaller than the corresponding ones of the SPP reflectivity and SPP transmissivity shown in Fig. 4. Furthermore, the comparison of Figs. 4 and 6, for a particular thickness and dielectric interface, shows that $R>R_{R}$ and $T>T_{R}$, which indicates that reflected and transmitted SPP fields carry more energy than their radiation equivalents.

Figure 8 shows the film-thickness dependence of the reflected and transmitted power 


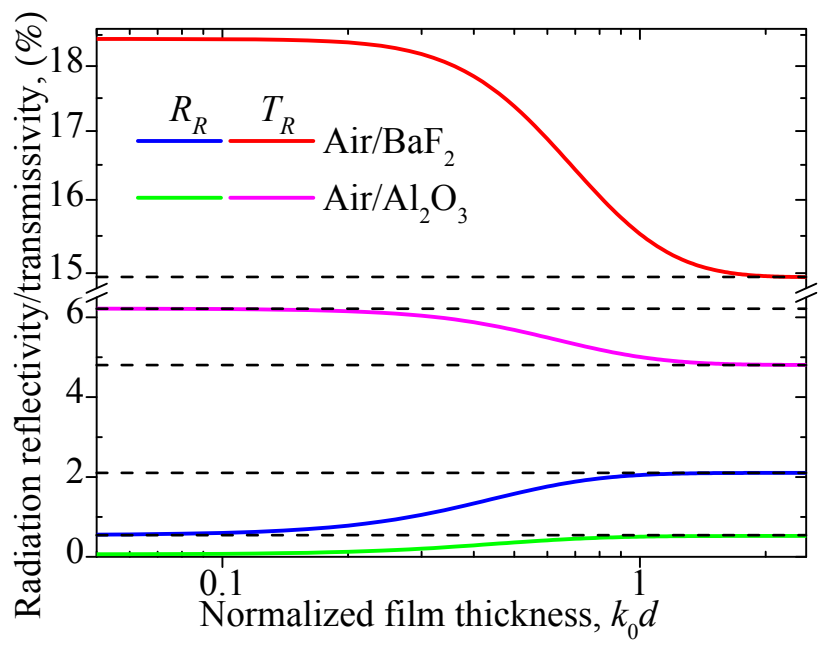

FIG. 7. Reflectivity and Transmissivity of the radiation fields generated by a SPP propagating along the surface of a $\mathrm{SiO}_{2}$ film and crossing a dielectric interface, as a function of the normalized film thickness. Calculations were done for two dielectric interfaces of $\mathrm{Air} / \mathrm{BaF}_{2}$ and $\mathrm{Air} / \mathrm{Al}_{2} \mathrm{O}_{3}$, and with the data used in Fig. 2.

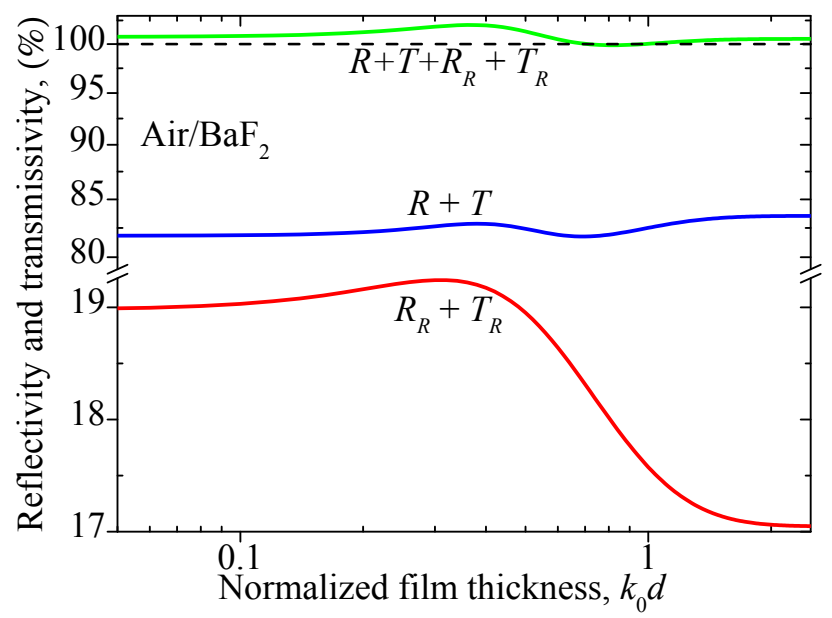

FIG. 8. Reflectivity+transmissivity involved in the SPP and radiation fields, as a function of the normalized film thickness. Calculations were performed with the results shown in Figs. 4 and 6.

fractions involved in the SPP $(R+T)$ and radiation $\left(R_{R}+T_{R}\right)$ fields, as well as their overall sum, for the Air $/ \mathrm{BaF}_{2}$ dielectric interface. The fact that $R+T>>R_{R}+T_{R}$ means that the major part of energy of the incident SPP is distributed between the reflected and trasmitted SPPs, while less than $20 \%$ of its energy goes to the radiation fields. Note that $R+T+R_{R}+T_{R} \approx 1$, which satisfies quite well the principle of conservation of energy, with a deviation of less than $2 \%$ that occurs at $k_{0} d=0.36(d=514 \mathrm{~nm})$. This deviation reduces 
to less than $0.5 \%$ for the dielectric interface of $\mathrm{Air} / \mathrm{Al}_{2} \mathrm{O}_{3}$, due to its smaller permittivity mismatch than the one of the Air $/ \mathrm{BaF}_{2}$ interface. It is therefore clear that, when the permittivities of the dielectric media are of the same order of magnitude, the proposed formalism is able to analytically describe with good accuracy the reflection and transmission of both the SPP and radiation fields generated at a dielectric interface. Given that Eqs. (15) and (20) have been derived assuming that the permittivivities of the dielectric media are not so dissimilar, in general, this simple approach is expected to yield deviations higher than $2 \%$, from the conservation of energy, as the difference of permittivities of the dielectric media increases to values larger than the unity $\left(\varepsilon_{3}-\varepsilon_{2}>1\right)$. In these cases, Eqs. (15a)-(15d) are not longer suitable and the determination of the reflection and transmission coefficients is much more complicated due to the presence of the radiation modes, as established by Eqs. (12) and (13), which still hold and can be conveniently solved by means of a numerical approach.

\section{CONCLUSIONS}

The reflection and transmission of a surface phonon-polariton propagating along the surface of a thin film of $\mathrm{SiO}_{2}$ and crossing the interface of two dielectric media has been analysed by means of an analytical approach based on the expansion of the electrical and magnetic fields in terms of normal modes. Fresnel-like formulas for the reflectivity and transmissivity of both the polariton and radiation fields generated at the dielectric interface have been explicitly determined. For the dielectric interfaces of air $/ \mathrm{BaF}_{2}$ and air $/ \mathrm{Al}_{2} \mathrm{O}_{3}$, it has been shown that: i) The polariton reflectivity (transmissivity) decreases (increases) as the film thickness increases, while its radiation equivalent follows the opposite behavior. ii) In the polariton and radiation fields, the transmissivity is significantly more sensitive than the reflectivity to the changes on the permittivity mismatch of the dielectric interface. iii) The reflectivity and transmissivity of the radiation fields are smaller than their polariton counterparts, which together account for around $82 \%$ of the energy of the incident surface phonon-polariton. The proposed formalism accurately fulfils the principle of conservation of energy and could be used for quantifying the polariton energy and radiation losses at a dielectric interface. 
[1] H. J. Bakker, S. Hunsche, and H. Kurz, Rev. Mod. Phys. 70, 523 (1998).

[2] N. Dahan, A. Niv, G. Biener, V. Kleiner, and E. Hasman, Opt. Lett. 33, 3195 (2005).

[3] B. Neuner, D. Korobkin, C. Fietz, D. Carole, G. Ferro, and G. Shvets, Opt. Lett. 34, 2667 (2009).

[4] D. Z. A. Chen, A. Narayanaswamy, and G. Chen, Phys. Rev. B 72, 155435 (2005).

[5] D. Z. A. Chen and G. Chen, Front. Heat Mass Transfer 1, 023005 (2010).

[6] J. Ordonez-Miranda, L. Tranchant, T. Antoni, Y. Chalopin, and S. Volz, J. Appl. Phys. 115, 054311 (2014).

[7] J. Ordonez-Miranda, L. Tranchant, B. Kim, Y. Chalopin, T. Antoni, and S. Volz, Appl. Phys. Express 7, 035201 (2014).

[8] D. Z. A. Chen and G. Chen, Appl. Phys. Lett. 91, 121906 (2007).

[9] P. Lambin, J. Vigneron, A. Lucas, P. Thiry, M. Liehr, J. Pireaux, R. Caudano, and T. Kuech, Phys. Rev. Lett. 56, 1842 (1986).

[10] J. Ordonez-Miranda, L. Tranchant, B. Kim, Y. Chalopin, T. Antoni, and S. Volz, Phys. Rev. Lett. 112, 055901 (2014).

[11] J. Ordonez-Miranda, L. Tranchant, T. Tokunaga, B. Kim, B. Palpant, Y. Chalopin, T. Antoni, and S. Volz, J. Appl. Phys. 113, 084311 (2013).

[12] M. Francoeur, M. P. Menguc, and R. Vaillon, Appl. Phys. Lett. 93, 043109 (2008).

[13] P. O. Chapuis, M. Laroche, S. Volz, and J. J. Greffet, Appl. Phys. Lett. 92, 201906 (2008).

[14] K. Torii, M. Ono, T. Sota, T. Azuhata, S. F. Chichibu, and S. Nakamura, Phys. Rev. B 62, $10861(2000)$.

[15] P. O. Chapuis, S. Volz, C. Henkel, K. Joulain, and J. J. Greffet, Phys. Rev. B 77, 035431 (2008).

[16] N. Ocelic and R. Hillenbrand, Nature Mater. 3, 606 (2004).

[17] H. C. Kim and X. Cheng, Opt. Lett. 35, 3748 (2010).

[18] J. J. Greffet, R. Carminati, K. Joulain, J. Mulet, S. Mainguy, and Y. Chen, Nature 416, 61 (2002).

[19] T. Taubner, F. Keilmann, and R. Hillenbrand, Nano Lett. 4, 1669 (2004). 
[20] E. Rousseau, A. Siria, G. Jourdan, S. Volz, F. Comin, J. Chevrier, and J. Greffet, Nature Photonics 3, 514 (2009).

[21] D. Z. A. Chen, R. Hamam, M. Soljacic, J. D. Joannopoulos, and G. Chen, Appl. Phys. Lett. 90, 181921 (2007).

[22] J. L. Gall, M. Olivier, and J. J. Greffet, Phys. Rev. B 55, 10105 (1997).

[23] F. Marquier, K. Joulain, and J. J. Greffet, Opt. Lett. 29, 2178 (2004).

[24] P. B. Catrysse and S. H. Fan, Phys. Rev. B 75, 075422 (2007).

[25] G. I. Stegeman, A. A. Maradudin, and T. S. Rahman, Phys. Rev. B 23, 2576 (1981).

[26] G. I. Stegeman, A. A. Maradudin, T. P. Shen, and R. F. Wallis, Phys. Rev. B 29, 6530 (1984).

[27] A. A. Maradudin, R. F. Wallis, and G. I. Stegeman, Prog. Surf. Sci. 33, 171 (1990).

[28] G. Stegeman, A. A. Maradudin, and T. S. Rahman, Phys. Rev. B 23, 2576 (1981).

[29] C. Yeh and F. I. Shimabukuro, The Essence of Dielectric Waveguides (Springer, New York, 2008).

[30] J. D. Jackson, Classical electrodynamics (New York, Wiley, 1998).

[31] V. Shevchenko, Continuous transitions in open waveguides (Golem Press, Boulder, Colorado, 1971).

[32] R. F. Oulton, D. F. P. Pile, Y. Liu, and X. Zhang, Phys. Rev. B 76, 035408 (2007).

[33] A. Voronko, L. Klimova, and G. Shkerdin, Solid State Commun. 61, 361 (1987).

[34] E. D. Palik, Handbook of Optical Constants of Solids (Academic Press, Orlando, Florida, 1985). 


\section{APPENDIX}

\section{A. Orthogonality of modes}

Let us consider that $\left(e_{n}, h_{n}\right)$ and $\left(e_{m}, h_{m}\right)$ are two different modes propagating along the system shown in Fig. 1 with $\varepsilon_{3}=\varepsilon_{2}$, they then satisfy Eqs. (1a) and (1c)

$$
\begin{gathered}
\frac{\partial^{2} h_{n}}{\partial x^{2}}-p_{n}^{2} h_{n}=0 \\
\frac{\partial^{2} h_{m}}{\partial x^{2}}-p_{m}^{2} h_{m}=0 .
\end{gathered}
$$

We can see that Eqs.(22a) and (22b) define a classical Sturm-Liouville problem, and hence the orthogonality of the $n^{t h}$ and $m^{t h}$ modes can be easily proved multiplying Eq. (22a) and the complex conjugate of Eq. (22b) by $h_{m}^{*}$ and $h_{n}$, respectively. The subtraction of the resulting equations yields

$$
\left(p_{n}^{2}-\left(p_{m}^{2}\right)^{*}\right) h_{n} h_{m}^{*}=\frac{\partial}{\partial x}\left(h_{m}^{*} \frac{\partial h_{n}}{\partial x}-h_{n} \frac{\partial h_{m}^{*}}{\partial x}\right) .
$$

By using Eqs. (1a) $(e=(\beta / \omega \varepsilon) h)$ and $(1 \mathrm{~b})\left(e_{z}=(1 / i \omega \varepsilon) \partial h / \partial x\right)$ for each mode and integrating both sides of Eq.(23), we obtain the following

$$
\frac{p_{n}^{2}-\left(p_{m}^{2}\right)^{*}}{i \beta_{n}} \int_{-d}^{\infty} e_{n} h_{m}^{*} d x=\left[e_{z n} h_{m}^{*}+\frac{\varepsilon^{*}}{\varepsilon} e_{z m}^{*} h_{n}\right]_{-d}^{\infty} .
$$

The right-hand side of Eq.(24) can be evaluated and simplified by splitting it for positions inside the thin film $(-d<x<0)$ and the dielectric medium $(x>0)$, applying the corresponding boundary conditions at $x=-d$ and $x=0$, and taking into account the disappearance of the fields at $x=\infty$. The final result is

$$
\frac{p_{n}^{2}-\left(p_{m}^{2}\right)^{*}}{i \beta_{n}} \int_{-d}^{\infty} e_{n} h_{m}^{*} d x=\left.\left(\frac{\varepsilon_{1}^{*}}{\varepsilon_{1}}-\frac{\varepsilon_{2}^{*}}{\varepsilon_{2}}\right) e_{z m}^{(1) *} h_{n}^{(1)}\right|_{x=-d} .
$$

Equation (25) thus indicates that when the permittivities of the thin film and the dielectric medium are real, the integral in its left-hand side vanishes

$$
\int_{-d}^{\infty} e_{n} h_{m}^{*} d x=0
$$

for any $n \neq m$. This orthogonality relation holds for any combination of SPP modes as well as for an inner product between SPP and radiation modes. Taking into account the definition of the Poynting vector in Eq.(19), Eq.(26) establishes that the power cannot be 
transferred among different modes in lossless media. Even though real materials always exhibit losses, these becomes negligible at certain excitation frequencies, as is the case of $\mathrm{SiO} 2$ and $\mathrm{SiC}[11,34]$. This is the reason why in this work, we are considering lossless media, for which Eq. (26) is valid. On the other hand, the normalization conditions of the SPP (Eq. (6a)) and radiation (Eq. (8a)) modes are straightforward to determine by direct integration of the modes in Eqs. (5) and (7), respectively.

\section{B. Solution of the SPP dispersion relation}

By writing the in-plane wave vector as $\beta=\sqrt{\varepsilon} k_{0}$, the transverse wave vectors $p_{n}=$ $\sqrt{\varepsilon-\varepsilon_{n}} k_{0}$ and the SPP dispersion relation in Eq. (4) reads

$$
\frac{\sqrt{\varepsilon-\varepsilon_{2}}}{\varepsilon_{2}}+\frac{\sqrt{\varepsilon-\varepsilon_{1}}}{\varepsilon_{1}} \tanh \left(\sqrt{\varepsilon-\varepsilon_{1}} \lambda\right)=0,
$$

where $\lambda=k_{0} d$ is the normalized film thickness. Considering that $\lambda<1$, which is the case of interest to enhance the SPP energy transport [11], Eq.(27) can be solved through perturbation theory. For an approximation up to $\lambda^{4}$, Eq. (27) takes the form

$$
\frac{\sqrt{\varepsilon-\varepsilon_{2}}}{\varepsilon_{2}}+\frac{\varepsilon-\varepsilon_{1}}{\varepsilon_{1}} \lambda\left(1-\frac{\varepsilon-\varepsilon_{1}}{3} \lambda^{2}\right)=0,
$$

which indicates that the effective permittivity $\varepsilon$ of the system has the following expansion

$$
\varepsilon=\varepsilon_{2}+\varepsilon^{(2)} \lambda^{2}+\varepsilon^{(4)} \lambda^{4}
$$

The combination of Eqs. (29) and (30) yields the following values for the parameters $\varepsilon^{(2)}$ and $\varepsilon^{(4)}$

$$
\begin{gathered}
\varepsilon^{(2)}=\varepsilon_{2}^{2}\left(1-\frac{\varepsilon_{2}}{\varepsilon_{1}}\right)^{2}, \\
\varepsilon^{(4)}=2 \varepsilon_{1}\left(1-\frac{\varepsilon_{2}}{\varepsilon_{1}}\right)\left[\frac{1}{3}-\left(\frac{\varepsilon_{2}}{\varepsilon_{1}}\right)^{2}\right] \varepsilon^{(2)} .
\end{gathered}
$$

Equations (24) and (25) are valid for any complex permittivity $\varepsilon_{1}$ of the film. For a lossless film ( $\varepsilon_{1}$ is a real number), however, these equations hold for $\varepsilon_{1}<0$ only. This is the condition

of existence and propagation of SPPs along very thin films $(\lambda<<1)$, which differs from the well known constraint $\varepsilon_{1}<-\varepsilon_{2}$, valid for thick films $(\lambda>>1)$. 